\title{
Perspectiva estudiantil sobre el plan de estudios de la carrera de Sociología de la Universidad Nacional de Costa Rica
}

Student's perspective of the study plan of the Sociology career of the Universidad Nacional of Costa Rica

\author{
Volumen 22 Número 1 \\ Enero - Abril \\ pp. 1-26
}

\author{
Diego Chaverri Chaves \\ María José Redondo Ríos \\ Daniel Ruiz Delgado
}

Citar este documento según modelo APA

Chaverri Chaves, Diego., Redondo Ríos, María José., y Ruiz Delgado, Daniel. (2022). Perspectiva estudiantil sobre el plan de estudios de la carrera de Sociología de la Universidad Nacional de Costa Rica. Actualidades Investigativas en Educación, 22(1), 126. Doi. https://doi.org/10.15517/aie.v22i1.47467 


\title{
Perspectiva estudiantil sobre el plan de estudios de la carrera de Sociología de la Universidad Nacional de Costa Rica
}

Student's perspective of the study plan of the Sociology career of the Universidad Nacional of Costa Rica

\author{
Diego Chaverri Chaves ${ }^{1}$ \\ María José Redondo Ríos ${ }^{2}$ \\ Daniel Ruiz Delgado ${ }^{3}$
}

Resumen: Este artículo muestra una investigación cuantitativa sobre el plan de estudios de la Escuela de Sociología de la Universidad Nacional, la cual emprendió la actualización de su currículo escolar; para tal efecto se tomaron las valoraciones por parte de la comunidad estudiantil que cursaba de tercero hasta sexto año de la carrera, para un total de 137 estudiantes. Dicho análisis se basó en un instrumento que abordó las cinco áreas del plan de estudio, a saber: 1) Realidad Social, 2) Teoría Sociológica, 3) Epistemología y Metodología, 4) Técnica e Instrumental y 5) Optativa; medidas a través de una escala Likert y una pregunta abierta para capturar otros aspectos afines. Los principales hallazgos resaltan que el rendimiento del programa de estudios no es homogéneo para sus estudiantes, pues muestra disparidades en las calificaciones asignadas, además, se observa que estudiantes de los últimos niveles están menos complacidos con su malla curricular, en comparación a estudiantes de niveles previos. Finalmente, en la pregunta abierta, la población estudiantil tiende a brindar sugerencias para mejorar, que disconformidades, lo que resalta la importancia de atender la calidad de la enseñanza y los procesos pedagógicos y andragógicos. A partir de los resultados obtenidos se identifican dificultades para separar la apreciación del plan de estudios de la valoración de la planta docente, también se recomienda; reestructurar y delimitar con mayor claridad las áreas de dicho plan, estimular y acompañar la formación didáctica del personal docente en la implementación de la carrera e integrar de manera más constante los aspectos teóricos y prácticos en la formación educativa.

Palabras clave: evaluación, estudiante universitario, plan de estudios, sociología.

Abstract: This article shows quantitative research about the study plan of the school of Sociology of the Universidad Nacional, which undertook the updating of its study plan; for this purpose, opinions were taken from the student community that went from the third to the sixth year of the degree; for a total of 137 students. This quantitative research, consisted of an instrument that addresses the five areas of the study plan, namely: 1) Social Reality, 2) Sociological Theory, 3) Epistemology and Methodology, 4) Technical and Instrumental and 5) Optional; measured through a Likert scale and an open question to capture other related aspects. The main findings highlight that the performance of the study plan is not homogeneous for its students, some areas have high marks, others do not, in addition, it is observed that students of the last levels are less pleased with their study plan, compared to students of previous levels. Finally, in the open question, the student population tends more to offer suggestions for improvement than disagreements, highlighting the importance of attending the quality of teaching and the pedagogical and andragogical processes. Based on the results obtained, difficulties are identified in separating the study plan appreciation from the assessment of the teaching staff, it is also recommended to restructure and delimit with greater clarity the areas of the study plan, stimulate and accompany the didactic training of the teaching staff in the implementation of the career, and integrate more constantly the theoretical and practical aspects in the educational training.

Keywords: evaluation, university student, study plan, sociology.

\begin{abstract}
1 Académico de la Universidad Nacional, en la Escuela de Sociología, Heredia, Costa Rica. Máster en Sociología de la Universidad de Costa Rica. Dirección electrónica: saludsociologia@gmail.com. Orcid: https://orcid.org/0000-0003-0708-4289
\end{abstract}

2 Estudiante de Licenciatura de la Universidad Nacional, Heredia, Costa Rica. Bachiller en Sociología por la Universidad Nacional. Dirección electrónica: maryredondorios@gmail.com Orcid: https://orcid.org/0000$\underline{0002-9179-7101}$

${ }^{3}$ Estudiante de Licenciatura de la Universidad Nacional, Heredia, Costa Rica. Bachiller en Sociología por la Universidad Nacional. Dirección electrónica:danielruiz121296@gmail.com Orcid: https://orcid.org/0000$\underline{0003-4688-8538}$

Artículo recibido: 16 de junio, 2021

Enviado a corrección: 14 de setiembre, 2021

Aprobado: 6 de diciembre, 2021

Los contenidos de este artículo están bajo una licencia Creative Commons 


\section{Introducción}

La carrera de Sociología, perteneciente a la Facultad de Ciencias Sociales de la Universidad Nacional de Costa Rica (UNA), a partir de la actualización de su plan de estudios en el período 2018-2021 se realizó un proceso de consulta a sus estudiantes sobre currículo escolar, el cual se llevó a cabo en el año 2019, de allí surge el objetivo principal de este estudio: identificar la percepción estudiantil sobre los aciertos y desaciertos de la malla curricular y obtener también críticas al respecto y alternativas para su mejora. Este proceso de consulta fue sustentado e implementado desde la Escuela de Sociología ubicada dentro del campus Omar Dengo en Heredia; involucrando la participación de docentes y primordialmente estudiantes.

Los resultados de este esfuerzo investigativo permitieron la elaboración del presente artículo, dando relevancia a la revisión de antecedentes, los cuales muestran una gran coincidencia en el protagonismo de la población estudiantil en las dinámicas de enseñanzaaprendizaje y el giro hacia modelos educativos que centran el proceso en el estudiantado, resultando decisivo levantar información sobre la perspectiva estudiantil. En este artículo se exponen antecedentes y referentes teóricos, la metodología y posteriormente los principales hallazgos de este trabajo, así como algunas consideraciones finales y sugerencias para introducir mejoras, tanto en procesos de consulta como en mallas curriculares, pensadas para el caso específico pero extensibles a otras carreras universitarias.

\section{Antecedentes y referentes teóricos}

No hay inconveniente en sostener que la evaluación de carreras universitarias es fundamental y un componente primordial para la toma de decisiones sobre la necesidad de modificar su orientación disciplinaria, actualizar o ampliar contenidos e innovar en prácticas académicas entendiendo que los procesos formativos requieren de una valoración constante (Segura, 2009). Sobre esto, el rol de los planes de estudio es central (Roldán, 2005) y puede considerarse (a propósito de la consulta que se aborda en este escrito) no sólo el criterio del estudiantado, sino también del personal académico y administrativo, personas egresadas, personas empleadoras y/o co-trabajadoras. En este sentido integrar cada una de las cuáles tiene diferentes apreciaciones de la experiencia del aporte disciplinar, así como la puesta en práctica de sus competencias, es decir, cómo se aplican: "El conjunto de aprendizajes significativos y útiles para el desempeño productivo en el trabajo, en los cuales las actitudes, 
los conocimientos y las destrezas adquiridas van a permitir el desarrollo exitoso de las tareas" (Ureña y Ureña, 2016, p.13).

Claro está, para establecer los cambios en un plan de estudio se debe realizar un trabajo que considere la fundamentación teórica, metodológica y andragógica de la carrera, requisitos, perfil de la planta docente, recursos didácticos e infraestructurales y otro vasto conjunto de criterios que en su conjunto permitirían justificar la pertinencia de dicha oferta escolar (Umaña et al., 2014). De tal modo, para esta investigación se procura tener un acercamiento al conjunto de la población estudiantil, sabiendo que se trata de personas con procedencias sociales heterogéneas, con divergencias en sus proyectos de vida e incluso en sus prioridades y preferencias respecto de una carrera como Sociología (Sibaja, 2020), por eso se toma cautela y sólo se derivarán reflexiones sobre los procesos de consulta a estudiantes y algunas sugerencias para la carrera de Sociología de la Universidad Nacional que se analiza, entendiendo las limitaciones de informantes con sus prerrogativas y expectativas diversas, pero miembros integrantes de la comunidad universitaria, que por tanto, brindan elementos no sólo de evaluación, sino también de (auto)evaluación (Mora, 2004).

Algunos estudios pueden preferir una evaluación de la formación a partir de las competencias y capacidades orientadas a las necesidades del sector empleador (Ureña y Ureña, 2016) o según la gestión y administración de los servicios escolares (Vera, 2018). Sin embargo, en esos casos, e incluyendo el estudio realizado en la Escuela de Sociología de la UNA, es muy complejo separar la evaluación de la calidad de una carrera o de la calidad de las personas docentes a cargo de la misma, pues se demanda de estas: conocimiento teórico, habilidades creativas, disposición ética y vocacional, métodos adecuados y el mantenimiento de relaciones interpersonales gratas (Alfaro y Alvarado, 2018; Rocha, 2013), entre otras cualidades que pueden ser más o menos distinguidas para diferentes carreras. En síntesis, la opinión de la población estudiantil, su perspectiva sobre aciertos y desaciertos de la enseñanza y el aprendizaje es también una herramienta para la evaluación educativa (Tirado, 2006).

Teóricamente, la coincidencia de estos antecedentes, nos señalan el interés en consultar a estudiantes puede suscribir una teoría de los procesos educativos centrada en la población aprendiente, como plantea Maryellen Weimer (2013) donde el estudiantado asume más tareas y retos, recibe instrucciones específicas para las habilidades y estímulo para la colaboración, con lo cual se le incentiva para que tome mayor control del propio proceso. En este caso específico la investigación considera otro aspecto adicional de esta teoría, pues la consulta brindó la oportunidad a cada discente para reflexionar sobre su propio proceso de aprendizaje, 
tanto en contenidos como en métodos (Weimer, 2013), sin ignorar las contradicciones y crisis de legitimidad que enfrentan las universidades, cada vez más sometidas a criterios comerciales transnacionales de eficiencia que suelen obstaculizar sus aspiraciones educativas (De Souza, 2005). Con estos referentes teóricos el artículo resalta la valoración del estudiantado, reconociendo su experiencia directa al sobrellevar la carrera, a través de este recurso se identifican algunos vacíos y aciertos de la malla curricular del Bachillerato y Licenciatura en Sociología de la UNA.

\section{Metodología}

El actual del plan de estudios de Sociología, disciplina con más de 40 años de existir en la Universidad Nacional de Costa Rica, tiene no menos de 15 años operando, de tal modo que ya múltiples generaciones lo han cursado completo y se han graduado, por tanto se hace oportuno contar con un estudio de la malla curricular a través del proceso de consulta a estudiantes como un pivote estratégico para comprender el desempeño de la carrera, sin menoscabo de otras valiosas informaciones como lo podría ser la apreciación que hacen personas egresadas, empleadoras, administrativas y académicas. El artículo opta por una metodología que desarrolla sus enunciados a partir de la perspectiva brindada por la población estudiantil.

El diseño curricular de carreras de educación superior debe considerar al estudiantado, de modo que los perfiles de egreso en los planes de estudio son relevantes para considerar la malla curricular y su interconexión con áreas, ejes u objeto de estudio así como las implicaciones para el estudiado (Toruño, 2019) y también para identificar los vínculos entre áreas del saber (Mora, 2013). En esta ocasión se optó por tomar los objetivos de la carrera para establecer una investigación cruzada con las áreas del plan de estudios, pensado justamente en la interpelación entre estos elementos y desde la perspectiva estudiantil, ya que: “(...) la capacidad de reflexionar del alumno sobre lo que ha conseguido y aquello que no ha logrado es la piedra angular de un gran número de enunciados de competencias" (San Martín et al., 2016, p. 11) asimismo, los instrumentos de encuesta permiten capturar una parte de la experiencia estudiantil, que se puede expresar en sus disposiciones y actitudes respecto de los procesos educativos en los que se ha involucrado (Hidalgo y Murillo, 2017).

A la par de los conocimientos que se puedan derivar, la actualización del plan de estudios necesita sumar otras fuentes testimoniales y también datos objetivos sobre su carrera como: recursos, infraestructura, matrícula, rendimiento, promoción y graduación, empleabilidad, entre 
tantos otros. De ningún modo se podría asumir que, aún siendo la valoración estudiantil central, sea suficiente para decidir todas las posibles modificaciones al plan de estudios o tener una mirada acabada sobre su impacto. Sin embargo, en correspondencia con el marco teórico y ante disconformidades con el plan de estudio se deben tomar medidas enfocadas cada vez más en el aprendizaje.

\subsection{Enfoque}

El enfoque de este trabajo fue cuantitativo, con alcance exploratorio, pues aunque se han realizado previamente consultas a estudiantes en la Escuela de Sociología, los resultados no han sido publicados. La información disponible es parcial y con algunos errores en la medición y en el levantamiento de datos, además, no evaluaban el plan de estudio sino aspectos generales de la carrera. De tal manera, se opta por un cuestionario que mediante escalas de Likert que mide los niveles de acuerdo con el grado de desempeño de los objetivos de la carrera y las áreas de formación en el plan de estudios, posteriormente se realiza un análisis descriptivo de los principales indicadores y se confeccionan índices para agrupar ítems y así establecer comparaciones entre estos.

\subsection{Unidades de análisis}

La población elegida se limitó a estudiantes regulares entre tercer y sexto año de la carrera, de quienes se espera, por el sistema de requisitos del plan de estudio, que ya hayan tenido contacto con al menos un curso de cada área e incluso haber aprobado hasta un 50\% del bachillerato, para esto se hizo un barrido en todos los grupos de una sola materia, distribuidos por los niveles, por lo cual se visitaron dos 2 grupos de tercer año de la carrera, dos grupos de cuarto año, dos grupos de quinto año de la carrera, y cuatro grupos de sexto año de la carrera, para un total de diez cursos visitados, de diferentes áreas y turnos horarios, procurando así aminorar sesgos de muestreo.

Todos los instrumentos fueron aplicados durante la segunda semana del primer ciclo lectivo del 2020, poco antes de las medidas de distanciamiento resultantes de la emergencia sanitaria por la sindemia global producto del coronavirus, para obtener una población total de 137 estudiantes participantes, con su consentimiento informado y en anonimato. De esta forma, se hizo un levantamiento de información aprovechando las ventajas de la concurrencia de estudiantes de la carrera en las aulas, teniendo acceso a un sector confiable y válido de la población. Con lo anterior no se pretende inferir conclusiones absolutas sobre la malla 
curricular, sino fundadas en la valiosa perspectiva estudiantil como se indicó en los referentes teóricos.

Aparte de los datos obtenidos se contaba con alguna información general de las Estadísticas Estudiantiles del Departamento de Registro, suscrito a la Vicerrectoría de Docencia de la Universidad Nacional, encargada del empadronamiento, matrícula y control administrativo de cada estudiante en toda la Universidad, institución que cada año recibe a más de tres mil nuevas personas estudiantes que se suman a la población de regulares y en conjunto superan un total de veinte mil estudiantes a nivel de grado y pregrado, aquí sólo se reseñan los datos específicos para el caso de la Escuela de Sociología.

Tomando en cuenta los últimos seis años, de donde procede mayormente la población encuestada, la carrera de Sociología en el 2013 tuvo 114 estudiantes matriculados en su primer ingreso, con una tendencia a la baja en los últimos años, por disminución del cupo, el año 2019 registró 90 estudiantes; en todos estos años se ha notado una proporción mayor de mujeres, la cual suele superar las dos terceras partes del total; igualmente mayoritaria es siempre la procedencia de estudiantes de colegios públicos, que en algunos años incluso supera los tres cuartos del total. Por su parte la cantidad de estudiantes regulares, a pesar de los cambios en ingreso y cupos, se ha mantenido algo más constante, en el 2013 había 407 matriculados, en el 2016 fueron 399, y en el 2019 eran 394 (Universidad Nacional, 2020).

\subsection{Técnicas de recolección de datos}

Para construir las escalas de medición de los cuestionarios se identificaron categorías centrales para definir los instrumentos, las cuales se cruzaron con los objetivos del plan de estudios y en pruebas piloto previas se capturó el énfasis que hace el estudiantado en la persona docente al evaluar los cursos, por eso se decidió desde el inicio que la consulta a estudiantes fuera de las áreas del plan de Estudio y no de cursos individuales, ya que en cada área, sin excepción, los cursos eran brindados por varias personas docentes (a pesar de los cambios entre generaciones y ciclos lectivos) así se disminuye la posibilidad de que la medición se desvié del plan de estudio y sus cursos y se dirija hacia su cuerpo académico. Sin embargo, no se evita el efecto colectivo de la calidad docente en la apreciación del plan de estudios, de hecho, una última pregunta abierta en el cuestionario que buscaba recoger cualquier aspecto residual, mostró en varias ocasiones referencia directa a docentes y sus competencias, lo cual reafirma que no se puede evitar el efecto sumado de cada profesional sobre el desempeño de esta carrera universitaria. 
En conciso, se indagó a través del cuestionario la apreciación total de las cinco áreas del plan de estudio a partir de seis objetivos derivados del mismo plan. De tal manera, las áreas del plan de estudio son: 1) Realidad Social, 2) Teoría Sociológica, 3) Epistemología y Metodología, 4) Técnica e Instrumental y 5) Optativa; para el detalle de los cursos de cada área, véase el anexo. Por su parte, los objetivos a evaluar se pensaron sobre una carrera que brindaría: 1) Formación científica rigurosa, 2) Destrezas para la solución de problemas, 3) Perspectiva crítica para la comprensión de la sociedad, 4) Reflexión sobre problemas sociales, 5) Contenidos vinculados con la actualidad y 6) Metodología apropiada para el aprendizaje.

Al cruzar las cinco áreas, con los seis objetivos, se obtuvieron treinta ítems, todos diseñados con frases afirmativas que se construyeron con la escala psicométrica de Likert ajustada al punto medio, con cinco opciones de consenso que varían así: Totalmente de acuerdo, Muy de acuerdo, Medianamente de acuerdo, Poco de acuerdo, Nada de acuerdo. Con esta fórmula se logra asertividad al medir justo el nivel de acuerdo y se evitan otras escalas sólo parcialmente ajustadas, que en un lado miden el consenso, luego el punto medio o neutral y en otro lado miden el disenso. Si bien se puede cuestionar que la escala sólo considera el "acuerdo", este puede tomar el valor extremo de "nada de acuerdo" y aplicado a frases en afirmativo permite tener precisión respecto del posicionamiento de cada informante, además, una pregunta abierta al final del instrumento (para todo tipo de comentarios residuales) también capturó aspectos negativos que muestran disensos y disconformidades con la carrera, sin embargo los comentarios obtenidos también mostraron alta conformidad con la carrera. Se estima que modificar la escala podría añadir confusión al instrumento y sin garantía de obtener resultados más fiables.

De características demográficas del estudiantado en el sondeo se requirió únicamente edad, sexo y año de la carrera, pues interesaba una apreciación del conjunto, sin pormenorizar en tipos de estudiantes y con estas tres variables explorar si hay variaciones dentro de las tendencias de los datos obtenidos, lo cuáles resultaron bastante homogéneos respecto del sexo y la edad, pero con una importante diferencia según el año de la carrera, como se expondrá más adelante.

En total el instrumento contó con treinta y seis variables (ver instrumento en el anexo 1) y se giraron instrucciones para que fuese aplicado por las mismas personas docentes de las materias o por un integrante del grupo investigador, además se incluía una tabla al inicio resumiendo los contenidos de cada área del plan de estudios, de modo que eso brinda mayor certeza de que cada estudiante tenga claridad de cuáles son las áreas y el subgrupo de cursos 
que está evaluando en cada oportunidad. Además, al final se dejó una pregunta abierta, para capturar observaciones pendientes, sobre la cual se realizó posteriormente una revisión manual de todas las respuestas, para establecer su respectiva codificación, ya que además de ser abierta era una pregunta que admitía respuestas múltiples. Este ítem funcionó muy bien, pues en efecto aparecieron aspectos que no atendían las demás variables y un total de 108 personas aportaron comentarios, sólo veintinueve se abstuvieron, lo cual muestra anuencia e interés en la temática de parte de la población de informantes, algo de vital importancia para este tipo de acercamientos empíricos y de modo congruente con la teoría señalada.

\subsection{Procesamiento de análisis}

Los datos obtenidos mediante los instrumentos se ingresaron en una base de datos con el subsecuente procesamiento estadístico. Así cada área tenía seis preguntas, para valorar el nivel de cumplimiento de cada objetivo, entonces se pasó a realizar conglomerados en los cuáles se sumaban todas las calificaciones de cada informante en las diferentes áreas, obteniendo cinco sumas, una del área de Realidad Social, del área de Teoría Sociológica, otra para el área de Metodología y Epistemología, una más para el área Técnica e Instrumental y la última para el área Optativa. Luego se realizaron del mismo modo sumatorias de las calificaciones de cada estudiante para los objetivos, dando con seis sumas, la primera para apreciar el aporte en la reflexión sobre problemas sociales y su impacto en las relaciones humanas, para valorar el vínculo de contenidos con la actualidad así como evaluar la adquisición de destrezas para resolver problemas, una más para considerar el nivel de rigor en la formación para la investigación científica social y las dos últimas para considerar la perspectiva crítica que brinda la carrera y para evaluar la metodología utilizada en los cursos.

Mientras los ítems se recogen en escalas de Likert según el nivel de acuerdo, las sumas de puntajes de áreas y de objetivos se subdividen igualmente en cinco calificaciones, que suponen una división equitativa en partes iguales de la escala, entre la calificación más alta posible y la calificación más baja posible, es decir, se trata también de calificaciones ponderadas, evitando que algunas tengan mayor posibilidad aritmética de capturar datos. Finalmente, esta reagrupación de los resultados se expone en una nueva escala, de tal modo la suma para cada área y para cada objetivo se etiqueta con valores de "Muy alto", "Alto", "Medio", "Bajo" y "Muy bajo", procurando simetría con la escala de valores utilizados en el cuestionario, que también era de cinco opciones. 


\section{Análisis y resultados del estudio: Las valoraciones del plan de estudios de la carrera de Sociología}

Con el levantamiento de información de la encuesta se obtuvo que la edad oscila entre los 19 y los 37 años, sin embargo, un $68,7 \%$ de la población encuestada se ubica entre los 20 y 23 años de edad es decir, se trata de estudiantes que en su mayoría absoluta se encuentran en el mismo rango etario que corresponde a quienes después de la educación secundaria optan inmediatamente por seguir una carrera universitaria. De modo congruente con los datos del Departamento de Registro, como se muestra en las Figuras 1 y 2, la aplicación de encuestas logró la participación mayoritaria de 103 mujeres, 30 hombres, 3 casos no identificados y 1 persona transgénero, además se logró una distribución equilibrada por niveles, con 39 de tercer año, 35 de cuarto año, 32 de quinto año y 30 de sexto año. En términos generales, el sondeo tuvo un funcionamiento adecuado, pues la gran mayoría de los instrumentos se complementaron en su totalidad, hay muy pocos datos perdidos o ítems sin respuesta y esto permite un análisis más íntegro del conjunto de los resultados.

Figura 1

Número de estudiantes participantes por género de Sociología de la UNA, Costa Rica. 2020

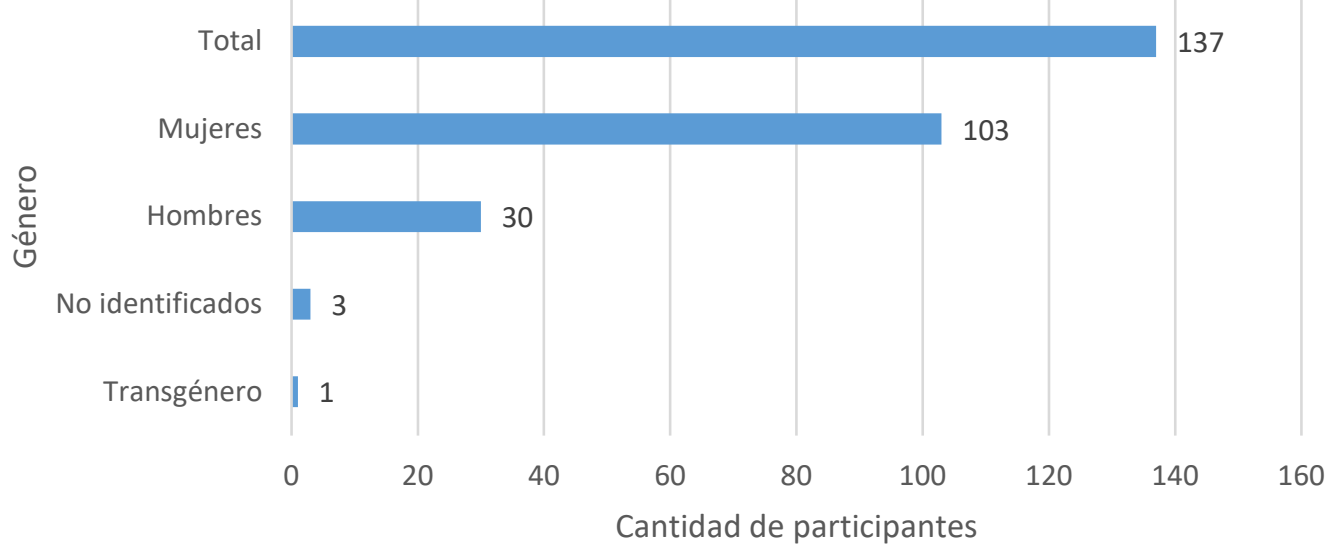

Fuente: Elaboración propia. 
Figura 2

Número de estudiantes participantes por nivel, de Sociología de la UNA, Costa Rica. 2020.

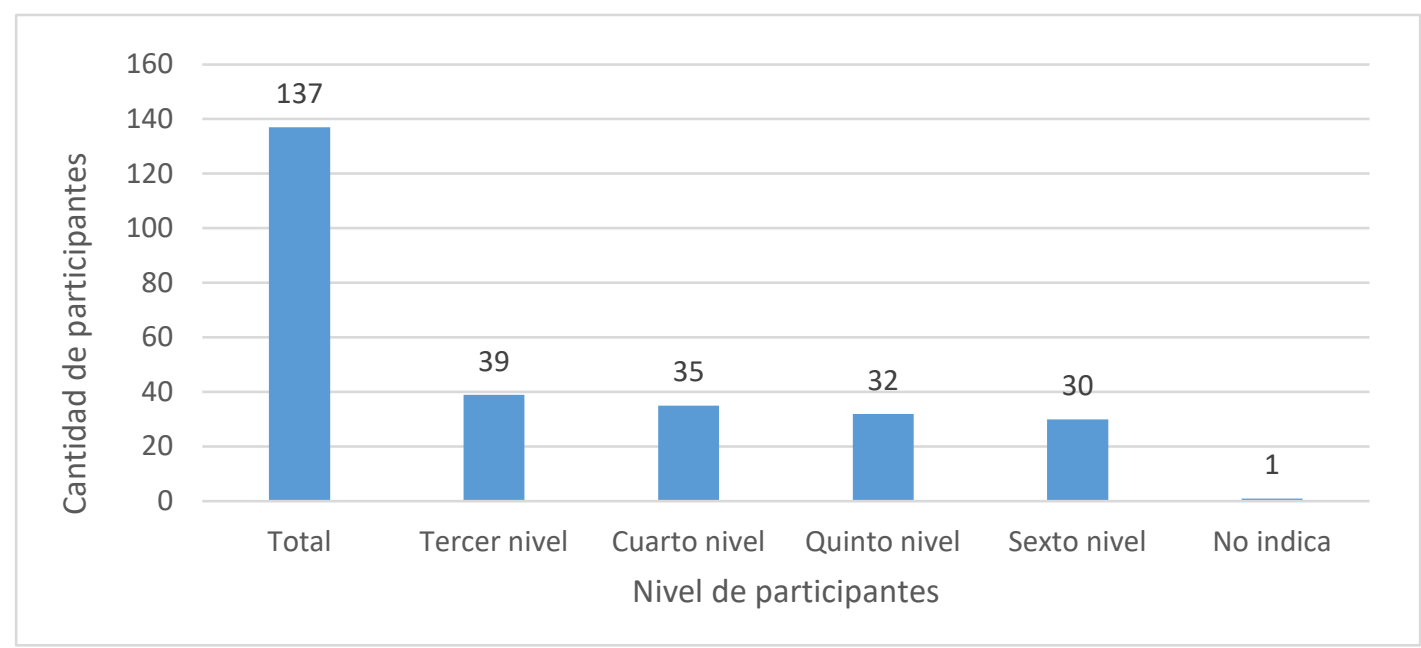

Fuente: Elaboración propia.

Como se aprecia en la Tabla 1, en el área de Realidad Social se obtuvieron los mayores niveles de aprobación, allí se considera alto o muy alto el aporte en el $47 \%$ de los casos, mientras que, en dos áreas, la de Teoría Sociológica y la de Metodología, la valoración alta y muy alta es de un 43,8\%, luego el área Optativa, con el mismo criterio, obtiene un $36,5 \%$, y el área Técnica disminuye a un $32,9 \%$ de valoración alta o muy alta. Las valoraciones bajas o muy bajas se presentaron en menor cantidad en todos los casos y hay también una fuerte concentración que oscila entre 33\% y 40\% para una valoración intermedia del aporte de las áreas del plan de estudios. También fue el área Técnica la que recibió la mayor cantidad de valoraciones bajas y muy bajas, con un total de 41 casos, mientras que el área de Realidad Social fue la menos afectada aquí, con sólo 16 casos, seguida por el área de Teoría, con 25 casos de valoración baja o muy baja. En resumen, el área de Realidad Social es la mejor calificada junto con el área de Teoría Sociológica, mientras que el área Técnica Instrumental es la que muestra el rendimiento más bajo. 
Tabla 1

Valoración en porcentajes por áreas del Plan de Estudios según estudiantes de Sociología de la UNA. Costa Rica, 2020.

\begin{tabular}{|l|c|c|c|c|c|c|}
\hline \multirow{2}{*}{ Área evaluada } & \multicolumn{6}{|c|}{ Valoración del aporte } \\
\cline { 2 - 7 } & Muy alto & Alto & Medio & Bajo & Muy bajo & No respondió \\
\hline Realidad Social & $3.7 \%$ & $43.8 \%$ & $40.9 \%$ & $10.9 \%$ & $0.7 \%$ & \\
\hline Teoría Sociológica & $5.8 \%$ & $38 \%$ & $38 \%$ & $15.3 \%$ & $2.9 \%$ & \\
\hline $\begin{array}{l}\text { Epistemología } \\
\text { Metodología* }\end{array}$ & $5.1 \%$ & $31.4 \%$ & $33.6 \%$ & $16.8 \%$ & $5.1 \%$ & $8 \%$ \\
\hline Técnica Instrumental & $1.5 \%$ & $31.4 \%$ & $37.2 \%$ & $22.6 \%$ & $7.3 \%$ & \\
\hline Optativa & $5.1 \%$ & $38.8 \%$ & $35 \%$ & $15.3 \%$ & $5.1 \%$ & $0.7 \%$ \\
\hline
\end{tabular}

Fuente: Elaboración propia.

Tomando ahora los seis objetivos se plasman los hallazgos en la Tabla 2; en la cual resalta que el más débil es respecto a la metodología para los cursos, pues sólo en el $26.3 \%$ de los casos se aprecia un desempeño alto o muy alto y en cambio una valoración baja o muy baja se observa en el orden del $27.7 \%$, esto llama la atención sobre la importancia de la enseñanza, sus estrategias y el impacto que esta tiene en la apreciación de la malla curricular de la carrera por parte del estudiantado.

En contraste, el objetivo mejor logrado es el aporte de la carrera para estimular la reflexión sobre los problemas sociales y su impacto en las relaciones humanas, con un $54.7 \%$ de calificación alta o muy alta, seguido del aporte en perspectiva crítica para la comprensión de la sociedad y sus conflictos que registró un $51.1 \%$ de valoraciones altas o muy altas. Un nivel de logro intermedio está en el cumplimiento de una formación científica rigurosa y la oferta de contenidos vinculados con la actualidad; en el primero, las dos valoraciones positivas suman un 39,4\%, en el segundo ascienden al 45.3\%. En el penúltimo puesto, la formación en destrezas para brindar soluciones a problemas específicos, obtuvo $32,1 \%$ de valoraciones positivas y un agregado de $21.9 \%$ de valoraciones negativas.

Como suele suceder con las escalas de Likert, hay una fuerte concentración de evaluaciones de nivel medio en todos los objetivos que oscila entre 34\% y 46\%, lo cual podría representar una situación ambivalente, según la cual esta parte del estudiantado no está disconforme con lo que recibe del plan de estudios, pero tampoco queda plenamente satisfecho. 
De modo sucinto, los dos objetivos que procuran brindar capacidad reflexiva sobre problemas sociales y ofrecer perspectiva crítica sobre los conflictos son los que mejor desempeño muestran, mientras que la apreciación de la metodología aplicada en los cursos y las destrezas que se obtienen para atender problemas concretos son los de más baja apreciación, por su parte la oferta actualizada y la formación científica para la investigación están en los puestos intermedios de apreciación.

Tabla 2

Valoración en porcentajes por objetivos del Plan de Estudios según estudiantes de Sociología de la UNA. Costa Rica, 2020.

\begin{tabular}{|l|l|l|l|l|l|}
\hline \multirow{2}{*}{ Objetivo evaluado } & \multicolumn{5}{|c|}{ Valoración del aporte } \\
\cline { 2 - 6 } & Muy alto & Alto & Medio & Bajo & Muy bajo \\
\hline Reflexión sobre problemas sociales & $8 \%$ & $46.7 \%$ & $34.3 \%$ & $9.5 \%$ & $1.5 \%$ \\
\hline Perspectiva crítica & $6.6 \%$ & $44.5 \%$ & $37.3 \%$ & $10.9 \%$ & $0.7 \%$ \\
\hline Contenidos vinculados con actualidad & $4.4 \%$ & $40.9 \%$ & $38 \%$ & $14.5 \%$ & $2.2 \%$ \\
\hline Formación científica & $0.7 \%$ & $38.7 \%$ & $43.8 \%$ & $14.6 \%$ & $2.2 \%$ \\
\hline Destrezas para brindar soluciones & $0.7 \%$ & $31.4 \%$ & $46 \%$ & $17.5 \%$ & $4.4 \%$ \\
\hline Metodología apropiada para los cursos & $2.2 \%$ & $24.1 \%$ & $46.4 \%$ & $21.7 \%$ & $5.6 \%$ \\
\hline
\end{tabular}

Fuente: Elaboración propia

Si se suman todas las valoraciones, de las cinco áreas y para los seis objetivos, tal como se hace abajo en la Tabla 3, se puede hacer un promedio de todas las valoraciones según cinco grupos de calificaciones: muy alto, alto, medio, bajo y muy bajo. Los resultados así obtenidos sólo mostraron un caso de una apreciación total muy baja, ninguno de una apreciación muy alta, mientras que en 49 ocasiones se obtuvo una valoración positiva, representando el $35.8 \%$ de los casos, la mayor cantidad la representa la valoración media, que con 64 casos representa un $46.7 \%$ y sólo el $16.1 \%$ de estudiantes dieron una calificación promedio baja, con 22 casos, en términos globales la valoración del plan de estudios es media, con tendencia a alta. Ahora bien, al hacer los cruces de variables entre edad, sexo y año de carrera con esta valoración promedio global, los datos mantenían las mismas tendencias en los dos primeros casos, es decir, la evaluación no varía por sexo o por edad de manera significativa, pero sí lo hace en cambio cuando se cotejan los puntajes con el año de la carrera que cursa el estudiantado, esto claramente mostró que hay una relación inversa directa entre el grado de apreciación de los aportes del plan de estudios y el nivel de carrera, al más alto nivel en la carrera más baja resultaba la calificación del plan de estudios. 
Tabla 3

Cantidad de estudiantes de Sociología de la UNA por nivel de carrera según valoración general del plan de estudios. Costa Rica, 2020.

Fuente: Elaboración propia.

\begin{tabular}{|l|l|l|l|l|l|}
\hline $\begin{array}{l}\text { Nivel de } \\
\text { carrera }\end{array}$ & Alta & Media & Baja & Muy baja & Total \\
\hline Tercero & 21 & 11 & 5 & 1 & 38 \\
\hline Cuarto & 12 & 17 & 6 & 0 & 35 \\
\hline Quinto & 12 & 17 & 3 & 0 & 32 \\
\hline Sexto & 4 & 19 & 7 & 0 & 30 \\
\hline No responde & 0 & 0 & 1 & 0 & 1 \\
\hline Total & 49 & 64 & 22 & 1 & 136 \\
\hline
\end{tabular}

El hallazgo anterior es negativo para la Escuela de Sociología, pues no sólo no mejora la percepción estudiantil de la oferta académica recibida conforme se avanza en la carrera, sino que incluso disminuye la valoración, esto es más grave si se considera que son justamente las personas estudiantes de años más avanzados (que poseen mayores conocimientos sobre el plan de estudios) quienes tienen una peor apreciación. Por su parte los casos extremos son atípicos, solo hay un caso de una valoración general muy baja y ningún caso de una valoración muy alta.

Como se observa en el cruce de variables, la población de tercer año es la que realiza mayor cantidad de calificación altas, con 21 casos, con sólo una calificación muy baja, esto puede deberse a que todavía en tercer año quede alguna persona con intenciones de pasarse de carrera (algo más esperable en primer año de la carrera), dicho de otra manera, es promisorio para la Escuela de Sociología que en cuarto, quinto y sexto año de carrera, ningún estudiante tenga una valoración promedio muy baja del plan de estudios.

Sin embargo, como se advertía, es en el último año de la carrera, donde estudiantes casi egresados y que ya empiezan a dedicarse a su trabajo final de graduación, tienen la menor cantidad de evaluaciones altas del plan de estudio y también la mayor cantidad de casos de evaluaciones bajas de este, lo cual es mucho más delicado si se recuerda que de toda la muestra la menor parte corresponde a estudiantes de este último nivel, es decir, mientras en tercer año se encuestaron 38 estudiantes, en sexto año fueron 30, esto implica que la calificación se agrava si se toman en cuenta ya no sólo las frecuencias, sino la proporción. Para expresarlo en porcentajes, si se toma únicamente a la población de tercer año de carrera un $55.2 \%$ tiene una valoración promedio alta y un $13.1 \%$ una valoración promedio baja del plan de estudios y en comparación, únicamente considerando ahora el estudiantado de último año de la carrera, sólo un $13.3 \%$ tiene una valoración promedio alta y un $25,3 \%$ una valoración 
promedio baja del plan de estudios, es decir, la tendencia se invierte. Claro está, para tener una apreciación más completa de este fenómeno aún habría que considerar el primer y segundo año de la carrera así como profundizar en los casos de mayor y menor conformidad con el plan de estudio y para apreciar detenidamente los extremos de estas tendencias.

En el caso de la pregunta final abierta a todo tipo y cantidad de comentarios, de las 108 personas que aportaron sus observaciones surgieron 239 comentarios en total, para esto obsérvese la Figura 3. Lo anterior se da porque el análisis valoraba también la extensión y complejidad de cada aporte, lo cual es concordante con los referentes conceptuales, desde los cuales se ha venido insistiendo en la riqueza de la experiencia estudiantil para comprender los alcances de una carrera.

De esta manera, cada estudiante realiza desde uno hasta cinco comentarios, distribuidos del siguiente modo: 31 estudiantes brindaron un único comentario, 39 estudiantes dieron dos comentarios, 28 estudiantes dieron tres y 10 estudiantes brindaron 4 o más comentarios. Dada esta multiplicidad de respuestas, después de su revisión individual y también colectiva, se definió primero agruparlos en 2 grandes vertientes: comentarios positivos que toman la forma de recomendaciones y comentarios negativos que expresan disconformidades, las cuales a su vez se subdividen en 6 tópicos sobre los cuales se pronunciaban y un último código que se hizo para casos atípicos. De tal modo, todas las respuestas se analizaron con 13 etiquetas de valor, 6 para comentarios positivos o sugerencias de cómo mantener o mejorar la calidad de la carrera, 6 para comentarios negativos con el estado actual y una última etiqueta para agrupar otras observaciones que no responden a ninguna de las anteriores.

En total, como se aprecia en la Figura 3, un número de 47 estudiantes plantearon 72 disconformidades mientras que 83 estudiantes plantearon 153 recomendaciones, en ese sentido,se muestra que la disposición del estudiantado hacia la carrera es mayoritariamente positivo y propositivo, pues incluso estudiantes que indican algún tipo de disconformidad en uno de sus comentarios, en el siguiente podían dar una recomendación, esto es congruente con los datos anteriores de la escala de Likert, que evidencian también una calificación mayoritariamente regular o alta de la malla curricular. Los comentarios, buenos o malos, se catalogaron en 6 tópicos: sobre cursos, sobre el mismo estudiantado, sobre un área del plan de estudios, sobre la carrera en general, sobre la administración de la Escuela y sobre aspectos pedagógicos o didácticos. El tópico con más comentarios fue aquel sobre cursos específicos, que obtuvo 28 disconformidades y 64 sugerencias, lo cual permite insistir, ahora desde la pregunta abierta, en el peso que la docencia tiene sobre la valoración del plan de 
estudios por el rol protagónico que tiene sobre un curso particular. Sobre esto mismo, es importante señalar que, de nuevo, en este tópico aparece reflejado el rol de académicos incluso de manera personalizada, hay que decir que tres docentes específicos fueron nombrados por sus errores o deficiencias, pero ninguna persona fue individualmente mencionada de manera positiva en los comentarios, el resto de observaciones eran respecto a la calidad docente en general, los contenidos, las evaluaciones y materiales utilizados en cursos singulares. Además, a este mismo asunto se le suman los comentarios sobre aspectos pedagógicos, con 14 recomendaciones y 16 disconformidades respecto del desempeño del cuerpo académico para acompañar los procesos de enseñanza y aprendizaje.

Es notable que la información que produce esta pregunta abierta concurre con lo que se observó en los antecedentes indicados arriba, las respuestas del estudiantado muestran que la evaluación de un plan de estudio no se puede considerar sólo por las escalas valorativas ante los objetivos en cada área del plan de estudio, el estudiantado también considera las competencias y destrezas, dilemas y posicionamientos éticos, su vocación o aptitudes, las relaciones interpersonales, así como los recursos e infraestructura.

Figura 3

Cantidad de comentarios sobre el plan de estudios según estudiantes participantes de Sociología de la UNA, Costa Rica. 2020

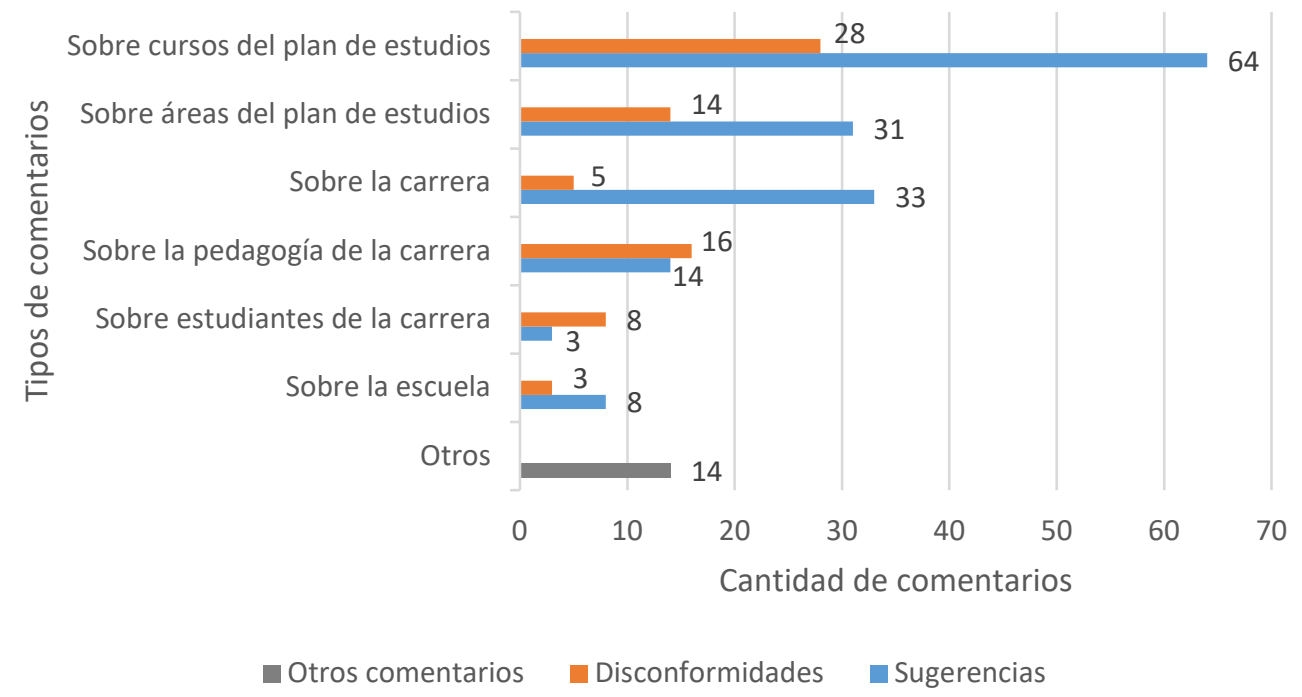

Fuente: Elaboración propia. 
Las dos temáticas con menos comentarios fueron sobre la Escuela como instancia administrativa, su capacidad de gestión o atención de problemas técnicos que incluyen los métodos sobre cómo elegir al profesorado a cargo de las varias materias, esto sólo obtuvo 3 comentarios negativos y 8 sugerencias. Mientras el tema del estudiantado, con observaciones sobre la propia motivación, técnicas de estudio, o la capacidad, el compromiso y solidaridad de compañeras y compañeros de carrera sólo tuvo 3 comentarios positivos y 6 negativos. El resto de las respuestas se distribuyen en comentarios sobre la carrera en general o sobre las mismas áreas del plan de estudio, de modo que permitieron profundizar en aspectos para tomar en cuenta en la actualización, así se tiene que en las áreas del plan de estudio hay 31 sugerencias y 14 disconformidades, mientras que sobre la carrera hay 33 recomendaciones y 5 disconformidades, entre los planteamientos destaca la necesidad de reforzar las formación en aspectos prácticos de la disciplina y promover teorías, métodos y técnicas actualizadas así como aplicables en escenarios concretos, con clases que brinden mayor profundización en contenidos, con explicaciones claras, mejorando el rigor, fomentando la criticidad y manteniendo espacios de participación y debate con estudiantes.

En muchos casos se hacían comentarios sobre los cursos y los que aparecían explícitamente mencionados eran en su mayoría del área Técnica y de Metodología, quiere decir que muchas de las sugerencias y disconformidades eran dirigidas hacia estos. Incluso con la última etiqueta, resaltó que las mismas áreas del plan de estudio están mal estructuradas y que dentro de una misma área se acota que hay heterogeneidad en los cursos allí recibidos, lo cual puede incidir en un sesgo del punto medio en los resultados presentados sobre las escalas de Likert.

\section{Conclusiones}

A diferencia de otros trabajos que buscan caracterizar a la población estudiantil que estudia una carrera (Sibaja 2020) o sus competencias (Ureña y Ureña, 2016) describir el perfil profesoral y calidad de sus docentes (Alfaro y Alvarado, 2018), consultar el parecer estudiantil sobre el desempeño docente (Rocha, 2013) o la calidad general de toda una carrera (Vera, 2018), este trabajo explora la apreciación que las personas estudiantes hacen de un plan de estudio, organizando la medición en áreas de la malla curricular y objetivos de la carrera, con esto se aprovecha su conocimiento directo de las dinámicas educativas y al mismo tiempo se les brinda oportunidad de reflexionar explícitamente sobre métodos y contenidos en su propio aprendizaje (Weimer, 2013). 
No se puede descuidar tampoco una perspectiva macro, para recordar que los desempeños escolares no dependen sólo de factores internos de los centros educativos, hay también estructuras y presiones de gobiernos nacionales y empresas transnacionales que limitan las posibilidades de modelos educativos democráticos (De Souza, 2005). Además las trayectorias educativas van en conjunto con otros procesos de socialización simultáneos que acompañan a las personas estudiantes a lo largo de su vida, con lo cual, varias dificultades escolares se pueden explicar no sólo por las deficiencias de un plan de estudio, sino también por las condiciones económicas, culturales e históricas de procedencia de cada estudiante e incluso la relación de los sistemas educativos con otros sistemas, estructuras e instituciones sociales (Chaverri, 2020).

Metodológicamente, la recolección de información, aun cuando se puede ver afectada por el ausentismo de estudiantes durante las fechas del trabajo de campo, hizo un barrido muy completo por cada uno de los cuatro niveles de la carrera considerados (de tercero a sexto), logrando una cantidad suficiente y bastante similar para cada generación, con lo cual se hizo posible la comparación por niveles, así la población de estudio resulta confiable. De esta forma, la encuesta representó una herramienta para capturar el testimonio del estudiantado sobre su malla curricular, permitiéndole opinar no solo sobre los cursos y sus áreas, sino generando también criterios sobre otros aspectos como la escuela, personal docente y administrativo, la población estudiantil y también sobre el mismo instrumento aplicado.

Adicionalmente, se debe tomar distancia crítica de los datos, no basta el testimonio que la población brinda a través de la encuesta, aún habría que profundizar en la observación del desempeño escolar del estudiantado, para contemplar, por ejemplo, la participación de estudiantes en actividades extracurriculares, su aprovechamiento de las oportunidades de aprendizaje que brindan la Universidad Nacional y la Escuela de Sociología e incluso cruzar la información con el rendimiento académico, para valorar cómo la apreciación que hacen del plan de estudio puede estar en alguna medida correlacionada con sus calificaciones a lo largo de la carrera e incluso sus relaciones interpersonales con el resto de la comunidad universitaria.

Se proponen aquí varias sugerencias derivadas del análisis de resultados; claro está, las mismas quedan sujetas al contraste con otras fuentes de información sobre el desempeño de esta unidad académica y su plan de estudios.

Primero, es importante señalar que desde varios análisis de la perspectiva estudiantil el desempeño docente es constantemente vinculado a la calidad del plan de estudios, por eso 
es prudente que toda actualización de dicho plan procure acompañarse de políticas que promuevan la constante evaluación, actualización y capacitación del cuerpo académico no sólo en contenidos, sino también en sus competencias educológicas para reforzar una enseñanza que no descuide el enfoque en el aprendizaje y por tanto en la persona aprendiente. Del mismo modo se debe mejorar en los criterios de reclutamiento de su planta académica, para que tomen mayor consideración de la calidad docente de cada quien y que las personas a cargo cuenten con perfiles académicos apropiados para los tipos de curso y muestren alto interés por la docencia, de manera tal que, consideren constantemente su impacto en el estudiantado y valoren formas de mejorar su entrega de la docencia, pues la calificación de la metodología docente en los cursos es la que obtuvo los más bajos resultados.

En consecuencia, es importante reconocer y estimular la participación estudiantil en procesos de consulta, como lo es el caso de estudiantes de la carrera de Sociología de la Universidad Nacional de quienes se obtuvo una perspectiva crítica sobre las y los encargados de implementar los procesos de enseñanza, los cursos y contenidos que se ofertan en la Unidad académica. En reiteradas ocasiones fue valorada de manera baja la labor docente en relación con el alcance de cursos y contenidos, por ello es necesario someter a un mayor análisis el papel que juega el cuerpo académico en la ejecución de un plan de estudios y proponer un monitoreo más constante sobre las formas en que son impartidos los cursos.

Se identificaron además reflexiones del estudiantado sobre sí mismos, las cuales evidencian el malestar que les provoca haber aprobado cursos con poca exigencia, de modo que expresan arrastrar carencias en su preparación profesional, especialmente en las áreas técnicas y prácticas, con esto se hace evidente, como se expuso al inicio, que este tipo de ejercicios de consulta sirven también como herramientas para la autoevaluación. Lo anterior es muestra de madurez escolar, se encuentran algunas personas capaces de cuestionar su propio aprendizaje, que son críticas del nivel académico logrado en su trayectoria por la carrera; no sólo se preocupan por aprobar los requisitos, también les interesa aprender las destrezas del oficio.

Al valorar conjuntamente el área de peor desempeño (Técnica-Instrumental) con el segundo objetivo menos logrado (brindar destrezas para la solución de problemas) es claro que hay un fallo en los cursos que deben dar mayor oportunidad para laboratorios, prácticas, trabajo de campo e incursión directa en escenarios concretos, se puede considerar entonces que los cursos prácticos orienten también su evaluación, contenidos y metodología en ese sentido; este déficit podría deberse a la necesidad de enfoques escolares con mayor atención 
en las personas aprendientes y allí son imprescindibles los cursos prácticos que permitan ejercitar a cada quien sus destrezas, acompañados por evaluaciones de proceso y no únicamente de resultado, así como realizadas individualmente, no sólo grupalmente.

Ahora bien, una dificultad peculiar que presentó este estudio es que, al procurar medir varios cursos dentro de una misma área, se descuida que dentro de este subconjunto de materias algunas son de tipo diferenciado entre sí y podrían reportar resultados muy distintos si se consideran de manera separada, quizá eso sume al sesgo del punto medio que se mencionó al inicio. La misma población estudiantil planteó esta discrepancia, por tanto se recomienda reestructurar el plan de estudios de modo más claro, ya que los cursos de Metodología y de Epistemología pueden tener una naturaleza muy diferente, además, el área Optativa no está claramente delimitada, pues en realidad suma cursos que son de todas las demás áreas, por eso se sugiere eliminar esta última, estrictamente como área y permitir que cada una de las otras áreas pueda ofertar sus respectivos cursos optativos, esto desde un punto de vista de diseño curricular tendría más congruencia. Así, se sugiere una malla curricular con cinco áreas: Sociedad, Teoría Sociológica, Metodología, Técnica-Instrumental y Epistemología.

Adicionalmente, es sensato considerar una malla curricular un poco más flexible en términos de requisitos y correquisitos de los cursos, para permitir al estudiantado mayor margen de maniobra al avanzar sobre el plan de estudios, esto apareció explícitamente mencionado por las personas encuestadas, así como ocasionalmente se recomendó la necesidad de actualizar contenidos dentro de los cursos, lo cual se vincula con los antecedentes de investigación que aquí se reseñaron, en tanto el contexto del ejercicio profesional y las posibilidades de empleo experimentan cambios frecuentemente, por eso es necesario someter cada tanto a investigación las carreras, sus planes de estudio y su impacto, ante lo cual, como ya se advirtió, la experiencia discente es imprescindible pero no suficiente; otros esfuerzos investigativos deben hacerse, para visualizar otras dimensiones y diferentes fuentes de información en la toma de decisiones sobre el diseño curricular.

Por último, considerando que el área de Realidad Social, seguida del área de Teoría Sociológica, son las dos mejor evaluadas y que los dos objetivos que apuntan a formar en análisis sobre las relaciones humanas y en perspectiva crítica sobre la sociedad son los de puntaje más elevado, la carrera de Sociología de la Universidad Nacional muestra madurez intelectual, por lo cual hay potencial para brindar soporte a las demás áreas y objetivos por mejorar, en tanto no se descuide y se busque aún fortalecer la ventaja que de momento ofrece 
la instrucción estudiantil centrada en la realidad concreta y acompañada de sustento teórico sociológico.

\section{Referencias}

Alfaro, Grettel., y Alvarado, Silvia. (2018). El perfil de profesores universitarios de universidades públicas y privadas en la carrera de Educación. Revista Actualidades Investigativas en Educación, 18(2) 1-21. https://doi.org/10.15517/aie.v18i2.33161

Chaverri, Diego. (2020). Dinámica de los grupos en educación. EUNED, Costa Rica.

De Sousa, Boaventura. (2005). La universidad en el siglo XXI. Para una reforma democrática y emancipadora de la universidad. UNAM, México: Centro de Investigaciones Interdisciplinarias en Ciencias y Humanidades.

Hidalgo, Nina., y Murillo, Javier. (2017). Las concepciones sobre el proceso de evaluación del aprendizaje de los estudiantes. REICE. Revista Iberoamericana sobre Calidad, Eficacia y Cambio en Educación, 107-128. https://www.redalyc.org/pdf/551/55149730007.pdf

Mora, Ana. (2004). La evaluación educativa: Concepto, períodos y modelos. Revista Actualidades Investigativas en Educación, https://www.redalyc.org/pdf/447/44740211.pdf

Mora, Rolando. (2013). Interconexiones de las diversas áreas del saber: elementos filosóficos, teóricos y prácticos. Praxis Revista de Filosofía, (70), 97-116. https://doi.org/10.15359/praxis.70.5

Rocha, Reynaldo. (2013). Escala de opinión de los Estudiantes sobre la Efectividad de la Docencia en Educación Superior. Revista Formación Universitaria, 6(6) 13-22. http://dx.doi.org/10.4067/S0718-50062013000600003

Roldán, Leda. (2005). Elementos para evaluar planes de estudio en la educación superior. Revista Educación, 29(1), 111-123. https://doi.org/10.15517/revedu.v29i1.2040

San Martín, Sonia., Jiménez, Nadia., y Sánchez, Estefanía. (2016). La evaluación del alumnado universitario en el espacio europeo de educación superior. Revista Aula Abierta, 44(1), 7-14. https://www.sciencedirect.com/sdfe/reader/pii/S0210277315000141/pdf

Segura, Mario. (2009). La evaluación de los aprendizajes basada en el desempeño por competencias. Revista Actualidades Investigativas en Educación, 9(2) 1-25. Doi 10.15517/aie.v9i2.9522

Sibaja, Irina. (2020). ¿Quiénes estudian sociología en la Universidad de Costa Rica? Revista de Ciencias Sociales, (170) 131-148. https://doi.org/10.15517/rcs.v0i170.47052 
Tirado, Felipe., Miranda, Alejandro., y Sánchez, Andrés. (2006). La opinión de los alumnos un recurso para la evaluación. Séptimo foro de Evaluación Educativa. San Luis Potosí. https://www.researchgate.net/publication/272295248 LA OPINION DE LOS ALUMN OS UN RECURSO PARA LA EVALUACION

Toruño, Cesar. (2019). Propuesta sistematizada para la evaluación de estructuras curriculares. El caso de la Universidad Técnica Nacional. Yulök Revista de Innovación Académica, 3(2) 29-43. https://doi.org/10.47633/yulk.v3i2\%20(julio\%20a\%20diciembre).200

Umaña, Ana., Calvo, Xinia., Fallas, Melba., Gómez, Gisselle., Jiménez, Laura., Soto, Suzanne., Salas, Natalia., y Tencio, Carolina. (2014). Guías para el diseño o rediseño curricular de planes de estudios de la Universidad Estatal a Distancia de Costa Rica. UNED, Costa Rica.

Universidad Nacional. (2020). Estadísticas estudiantiles de la Universidad Nacional. Vicerrectoría de Docencia, Departamento de Registro. UNA, Costa Rica. https://www.registro.una.ac.cr/see/

Ureña, Elvia., y Ureña, Viria (2016). Las competencias genéricas en la formación del estudiantado de la enseñanza del inglés en la universidad de costa rica: visión de docentes y estudiantes y su relación con las demandas del sector empleador. Revista InterSedes, 17(36) 118-151. https://doi.org/10.15517/isucr.v17i36.26977

Vera, Fernando. (2018). Percepción de estudiantes respecto a la calidad educativa y organizacional de la carrera de enfermería de una universidad privada chilena. Revista Electrónica Educare, 22(3), 1-25. http://dx.doi.org/10.15359/ree.22-3.1

Weimer, Maryellen. (2013). Learner-centered teaching: Five key changes to practice. Wiley, Estados Unidos. 


\section{Anexo}

Anexo 1. Instrumento para la evaluación del plan de estudios. Versión modificada para el formato de esta publicación.

\section{EVALUACIÓN DEL PLAN DE ESTUDIOS DE LA CARRERA DE SOCIOLOGÍA}

Presentación: Como parte de las actividades para la actualización del Plan de Estudios de la Carrera de Sociología de la Universidad Nacional, se le invita a participar en esta evaluación, la cual es totalmente anónima y para usos estrictamente académicos. De antemano agradecemos mucho su sincera colaboración.

NRC del curso en que se aplicó este instrumento

Edad en años cumplidos:
Sexo:

Número de instrumento

Por favor lea con detenimiento la distribución de cursos según áreas antes de responder el cuestionario.

\section{MALLA CURRICULAR DEL BACHILLERATO \& LICENCIATURA EN SOCIOLOGÍA POR}

$$
\text { ÁREAS }
$$

Cursos del área de REALIDAD SOCIAL (7 cursos): Taller de Realidad Social, Taller de Realidad Socio-Histórica I, Taller de Realidad Socio-Histórica II, Taller de Realidad SocioHistórica III, Proyecto de Graduación I, Proyecto de Graduación II, Proyecto de Graduación III.

Cursos del área de TEORÍA SOCIOLÓGICA (8 cursos): Introducción a la Sociología, Teoría Sociológica I, Teoría Sociológica II, Teoría Sociológica III, Teoría Sociológica Contemporánea I, Teoría Sociológica Contemporánea II, Taller de Análisis Sociológico, Seminario de debate Sociológico Actual.

Cursos del área de EPISTEMOLOGÍA Y METODOLOGÍA (9 cursos): Introducción a la Teoría del Conocimiento Sociológico, Construcción de Objetos Sociológicos, Perspectivas Metodológicas de las Ciencias Sociales I, Perspectivas Metodológicas de las Ciencias Sociales II, Perspectivas Metodológicas de las Ciencias Sociales III, Epistemología de las Ciencias Sociales, Sociología Inter y Transdisciplinariedad I, Sociología Inter y Transdisciplinariedad II, Sociología Inter y Transdisciplinariedad III.

Cursos del área TÉCNICA E INSTRUMENTAL (11 cursos): Técnicas de Investigación I, Técnicas de Investigación II, Estadística Social I, Estadística Social II, Taller de Análisis de Contenido, Taller de Diseño de Investigación, Práctica Profesional Supervisada, Taller de diseño, seguimiento y evaluación de proyectos I, Taller de diseño, seguimiento y evaluación de proyectos II, Taller sobre escenarios e indicadores sociales, Sistemas de Información Social.

Cursos del área OPTATIVA (3 cursos): Estadística multivariable, Técnicas de análisis espacial. Cursos o seminarios de autor (por ejemplo, Giddens, Marx, Durkheim, Weber, etc.). Cursos de sociologías de áreas específicas (e.g. sociología de la educación, género, ambiente, etc.). 
A continuación, se le presentan varias afirmaciones respecto a los cursos de cada área del Plan de Estudios, marque con $\mathbf{X}$ la opción que mejor refleje su parecer respecto de esa área específica a partir de su experiencia. Si tiene dudas respecto de cuáles cursos pertenecen a las áreas, revise de nuevo la malla curricular arriba. La escala valorativa va de 1 a 5 , donde cada número mide su apreciación de cada afirmación del siguiente modo: $(1)=$ Totalmente de acuerdo / (2) = Muy de acuerdo / (3) Medianamente de acuerdo / (4) Poco de acuerdo / (5) Nada de acuerdo.

\begin{tabular}{|c|c|c|c|c|c|}
\hline \multirow{2}{*}{ 1. CURSOS DEL ÁREA DE REALIDAD SOCIAL } & \multicolumn{5}{|c|}{ VALORACIÓN } \\
\hline & 1 & 2 & 3 & 4 & 5 \\
\hline \multicolumn{6}{|l|}{$\begin{array}{l}1.1 \text { "Los cursos del área de realidad social me han aportado } \\
\text { formación científica rigurosa, para el desarrollo de habilidades para } \\
\text { la investigación social" }\end{array}$} \\
\hline \multicolumn{6}{|l|}{$\begin{array}{l}1.2 \text { "Los cursos del área de realidad social me han aportado } \\
\text { destrezas para brindar soluciones a problemas sociales específicos" }\end{array}$} \\
\hline \multicolumn{6}{|l|}{$\begin{array}{l}1.3 \text { "Los cursos del área de realidad social me han aportado } \\
\text { perspectiva crítica, para la comprensión de la sociedad y sus } \\
\text { conflictos" }\end{array}$} \\
\hline \multicolumn{6}{|l|}{$\begin{array}{l}1.4 \text { "Los cursos del área de realidad social me han aportado reflexión } \\
\text { sobre problemas sociales, para entender su impacto en las } \\
\text { relaciones humanas" }\end{array}$} \\
\hline \multicolumn{6}{|l|}{$\begin{array}{l}1.5 \text { "Los cursos del área de realidad social me han aportado un } \\
\text { abordaje de contenidos vinculado con la actualidad" }\end{array}$} \\
\hline \multicolumn{6}{|l|}{$\begin{array}{l}1.6 \text { "Los cursos del área de realidad social se imparten con una } \\
\text { metodología apropiada para estimular mi aprendizaje" }\end{array}$} \\
\hline & \multicolumn{5}{|c|}{ VALORACIÓN } \\
\hline 2. CURSOS DEL ÁREA DE TEORÍA SOCIOLÓGICA & 1 & 2 & 3 & 4 & 5 \\
\hline $\begin{array}{l}2.1 \text { "Los cursos del área de teoría sociológica me han aportado } \\
\text { formación científica rigurosa, para el desarrollo de habilidades para } \\
\text { la investigación social" }\end{array}$ & & & & & \\
\hline $\begin{array}{l}2.2 \text { "Los cursos del área de teoría sociológica me han aportado } \\
\text { destrezas para brindar soluciones a problemas sociales específicos" }\end{array}$ & & & & & \\
\hline $\begin{array}{l}2.3 \text { "Los cursos del área de teoría sociológica me han aportado } \\
\text { perspectiva crítica, para la comprensión de la sociedad y sus } \\
\text { conflictos" }\end{array}$ & & & & & \\
\hline $\begin{array}{l}2.4 \text { "Los cursos del área de teoría sociológica me han aportado } \\
\text { reflexión sobre problemas sociales, para entender su impacto en las } \\
\text { relaciones humanas" }\end{array}$ & & & & & \\
\hline $\begin{array}{l}2.5 \text { "Los cursos del área de teoría sociológica me han aportado un } \\
\text { abordaje de contenidos vinculado con la actualidad" }\end{array}$ & & & & & \\
\hline $\begin{array}{l}2.6 \text { "Los cursos del área de teoría sociológica se imparten con una } \\
\text { metodología apropiada para estimular mi aprendizaje" }\end{array}$ & & & & & \\
\hline
\end{tabular}




\begin{tabular}{|c|c|c|c|c|c|}
\hline \multirow[b]{2}{*}{ 3. CURSOS DEL ÁREA DE EPISTEMOLOGÍA Y METODOLOGÍA } & \multicolumn{5}{|c|}{ VALORACIÓN } \\
\hline & 1 & 2 & 3 & 4 & 5 \\
\hline $\begin{array}{l}\text { 3.1"Los cursos del área de epistemología y metodología me han } \\
\text { aportado formación científica rigurosa, para el desarrollo de } \\
\text { habilidades para la investigación social" }\end{array}$ & & & & & \\
\hline $\begin{array}{l}\text { 3.2 "Los cursos del área de epistemología y metodología me han } \\
\text { aportado destrezas para brindar soluciones a problemas sociales } \\
\text { especificos" }\end{array}$ & & & & & \\
\hline $\begin{array}{l}\text { 3.3 "Los cursos del área de epistemología y metodología me han } \\
\text { aportado perspectiva crítica, para la comprensión de la sociedad y } \\
\text { sus conflictos" }\end{array}$ & & & & & \\
\hline $\begin{array}{l}3.4 \text { "Los cursos del área de epistemología y metodología me han } \\
\text { aportado reflexión sobre problemas sociales, para entender su } \\
\text { impacto en las relaciones humanas" }\end{array}$ & & & & & \\
\hline $\begin{array}{l}\text { 3.5 "Los cursos del área de epistemología y metodología me han } \\
\text { aportado un abordaje de contenidos vinculado con la actualidad" }\end{array}$ & & & & & \\
\hline $\begin{array}{l}\text { 3.6 "Los cursos del área de epistemología y metodología se imparten } \\
\text { con una metodología apropiada para estimular mi aprendizaje" }\end{array}$ & & & & & \\
\hline
\end{tabular}

\section{CURSOS DEL ÁREA TÉCNICA E INSTRUMENTAL}

\begin{tabular}{|c|c|c|c|c|}
\hline \multicolumn{5}{|c|}{ VALORACIÓN } \\
\hline $\mathbf{1}$ & $\mathbf{2}$ & $\mathbf{3}$ & $\mathbf{4}$ & $\mathbf{5}$ \\
\hline
\end{tabular}

4.1"Los cursos del área técnica e instrumental me han aportado formación científica rigurosa, para el desarrollo de habilidades para la investigación social"

4.2 "Los cursos del área técnica e instrumental me han aportado destrezas para brindar soluciones a problemas sociales específicos" 4.3 "Los cursos del área técnica e instrumental me han aportado perspectiva crítica, para la comprensión de la sociedad y sus conflictos"

4.4 "Los cursos del área técnica e instrumental me han aportado reflexión sobre problemas sociales, para entender su impacto en las relaciones humanas"

4.5 "Los cursos del área técnica e instrumental me han aportado un abordaje de contenidos vinculado con la actualidad"

4.6 "Los cursos del área técnica e instrumental se imparten con una metodología apropiada para estimular mi aprendizaje" 


\begin{tabular}{|c|c|c|c|c|c|}
\hline \multirow[b]{2}{*}{ 5. CURSOS DEL ÁREA OPTATIVA } & \multicolumn{5}{|c|}{ VALORACIÓN } \\
\hline & 1 & 2 & 3 & 4 & 5 \\
\hline $\begin{array}{l}5.1 \text { "Los cursos del área optativa me han aportado formación } \\
\text { científica rigurosa, para el desarrollo de habilidades para la } \\
\text { investigación social" }\end{array}$ & & & & & \\
\hline $\begin{array}{l}5.2 \text { "Los cursos del área optativa me han aportado destrezas para } \\
\text { brindar soluciones a problemas sociales específicos" }\end{array}$ & & & & & \\
\hline $\begin{array}{l}.3 \text { "Los cursos del área optativa me han aportado perspectiva } \\
\text { crítica, para la comprensión de la sociedad y sus conflictos" }\end{array}$ & & & & & \\
\hline $\begin{array}{l}5.4 \text { "Los cursos del área optativa me han aportado reflexión sobre } \\
\text { problemas sociales, para entender su impacto en las relaciones } \\
\text { humanas" }\end{array}$ & & & & & \\
\hline $\begin{array}{l}5.5 \text { "Los cursos del área optativa me han aportado un abordaje de } \\
\text { contenidos vinculado con la actualidad" }\end{array}$ & & & & & \\
\hline $\begin{array}{l}5.6 \text { "Los cursos del área optativa se imparten con una metodología } \\
\text { apropiada para estimular mi aprendizaje" }\end{array}$ & & & & & \\
\hline
\end{tabular}

6. Indique cualquier otro comentario que quiera usted hacer sobre el plan de estudios de esta carrera: 
Revista indizada en

sciplo redalyc satindex

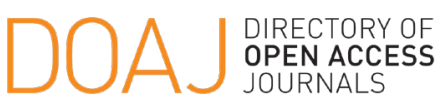

Distribuida en las bases de datos:

- Dialnet

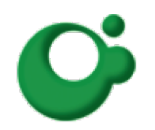
SHERPA/RoMEO REDIB

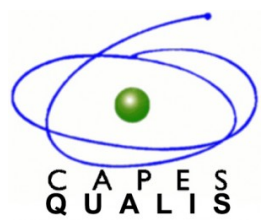

MIAR 